\title{
Predictors of therapeutic alliance in two treatments for adults with severe and enduring anorexia nervosa
}

\author{
Colleen Stiles-Shields ${ }^{1 *}$, Bryony H. Bamford ${ }^{2}$, Stephen Touyz ${ }^{3}$, Daniel Le Grange ${ }^{4,5}$, Phillipa Hay ${ }^{6}$ and Hubert Lacey ${ }^{7}$
}

\begin{abstract}
Background: Therapeutic alliance (TA) has been found to be a significant predictor of outcome for patients with severe and enduring anorexia nervosa (SE-AN), accounting for more variance than treatment type. To better understand how to promote TA for this population, the aim of the current study was to investigate predictors of TA in adults with SE-AN.
\end{abstract}

Methods: Participants were 63 adult females with SE-AN presenting to an outpatient, multi-site randomized controlled trial conducted at two clinical sites. Participants' perception of the quality of their therapeutic relationship, demographic information, and eating disorder symptomatology were assessed via interview and questionnaire measures.

Results: Baseline ratings of how successful participants believed treatment would be for them was the only variable to significantly predict early $(p=.01)$, mid $(p=.009)$, and late treatment alliance $(p=.03)$. No other variables investigated predicted the quality of patient rated TA at any point in treatment ( $p s>.57$ ).

Conclusions: Results suggest instilling hope in treatment outcome may enhance TA, and in turn, outcomes for patients with SE-AN in outpatient therapy.

Keywords: Therapeutic alliance, Anorexia nervosa, Cognitive behavioral therapy, Specialist supportive clinical management, Predictors

\section{Background}

Therapeutic alliance (TA) is a relational bond that develops between a patient and clinician via collaborative work and trust, as both endeavor to establish and accomplish treatment goals [1]. Cited as a critical nonspecific factor in the treatment of depression [2], TA has been shown to predict outcomes across a variety of treatments and disorders, including in chronic presentations [3-10]. In the treatment of eating disorders, TA is often considered an important element of interventions, but with mixed effect on treatment success [11-16].

TA has been shown to play a central role in preventing premature treatment drop-out in an inpatient population with anorexia nervosa (AN) $[17,18]$, with even initial impressions promoting treatment compliance [17]. Further, in a sample of adults with severe and enduring

\footnotetext{
* Correspondence: ColleenSS@u.northwestern.edu

${ }^{1}$ Department of Preventive Medicine, Northwestern University Feinberg

School of Medicine, Chicago, IL, USA

Full list of author information is available at the end of the article
}

anorexia nervosa (SE-AN), TA was found to be a significant predictor of eating disorder symptoms at end of treatment (EOT) and at follow-up [19]. Indeed, TA actually accounted for more of the variance in outcome than treatment type. As such, correlates of TA are a critical domain to explore for patients with SE-AN.

While TA is recognized as an important predictor of treatment engagement, less is known about the factors that may contribute to the development of a strong alliance in patients with AN, particularly those with a severe and enduring course of illness. Research within the field of anxiety and depression indicates that patients with more severe symptoms report poorer TA [20]; however, these associations between symptom severity and TA have not been replicated in adults with bulimia nervosa (BN) [21]. The quality of the therapeutic relationship has been noted as worse in individuals with chronic eating disorders [22], suggesting that duration of illness, or number of previous treatment episodes may adversely affect treatment engagement and TA. Additionally, when 
exploring psychotherapy processes, patient preconceptions and expectations regarding improvement have been found to be associated with stronger alliance and better overall treatment outcomes [23, 24]. This finding has been supported within a treatment trial for BN [21]. Further, there is recent evidence to suggest that positive early symptom change may be responsible for enhancing TA in patients with BN [11, 14]. Despite these findings, there is still a dearth of research investigating whether, and which, patient characteristics influence TA in SEAN. Greater understanding of patient characteristics that may influence therapeutic alliance and treatment engagement is needed to help clinicians identify specific treatment adaptations that may be needed to better tailor treatment to individual need.

The aim of this paper is to explore possible correlates of TA that may be present, irrespective of treatment or therapist variation. In line with previous research highlighted above, we hypothesized that characteristics associated with SE-AN (i.e., longer treatment duration, increased severity, higher previous treatment episodes, lower motivation and expectation of treatment success, and lower body mass index) would predict poorer participant-rated TA for adults with SE-AN.

\section{Methods}

This study is a secondary analysis of data from a randomized clinical trial (RCT) conducted at two clinical sites (The University of Sydney and St. George's Hospital, University of London), with a Data and Coordinating Center (The University of Chicago Medicine). The RCT compared the efficacy of Cognitive Behavioral Therapy for anorexia nervosa (CBT-AN) and Specialist Supportive Clinical Management (SSCM) for a sample of adults with SE-AN. Specific treatment effects have been analysed and are reported in the main outcome paper [25]. Participants received 30 individual, outpatient treatment sessions provided over eight months.

\section{Participants}

Recruitment of participants occurred from July 2007 to November 2010 through advertising to eating disorder clinics, clinicians, and generic websites.

Participants were eligible for randomization if they met the Diagnostic and Statistical Manual for Mental Disorders, $4^{\text {th }}$ Edition, Text Revision (DSM-IV-TR) [26] criteria for AN, excluding criterion D (amenorrhea); had an illness duration of at least 7 years; were at least 18 years of age; and were female. Exclusion criteria included presenting with a current manic episode or psychosis; current alcohol or substance abuse or dependence; significant current medical or neurological illness (including seizure disorder), with the exception of nutrition-related alterations that are impacted by weight; current engagement in psychotherapy and being unwilling to suspend such treatment for the duration of their participation in the study; and plans to move beyond commuting distance for the study site in the following 12 months or not living within commuting distance to the study site.

In compliance with the Institutional Review Boards (IRB) of the two intervention sites and the data and coordinating center, participants completed written informed consent prior to assessment. In addition to IRB approval, the trial was approved by the Human Research Ethics Committee (approval number 02-2007/9669) and was registered with the Australian New Zealand Clinical Trials Registry (ACTRN12607000440426).

\section{Treatments and therapists}

Participants were randomized to either CBT-AN or SSCM by a biostatistician independent from both intervention sites. Randomization was conducted using Ephron's biased coin approach, stratified within sites by: 1) subtype of illness (Restrictive and Binge-Purge) and 2) psychopharmacological medication status. Treatment occurred in outpatient settings at The University of Sydney and St. George's Hospital, University of London. Treatments and therapist characteristics are described in detail in previous papers $[25,27]$.

\section{Measures}

\section{Physical assessment}

Participants were weighed in light, indoor clothing with their shoes removed. Weight and height were measured by a trained research assistant using a calibrated digital or balance-beam scale and stadiometer, respectively, to calculate body mass index $\left(B M I=\mathrm{kg} / \mathrm{m}^{2}\right)$.

\section{Helping Relationships Questionnaire (HRQ)}

The HRQ measures the patient's perspective of the therapist-patient relationship via an 11 self-report items [28]. Items for the HRQ are rated on a 6-point likert scale, ranging from -3 ("Strongly feel it is not true") to +3 ("Strongly feel it is true"). Total scores, computed by summing all items, range from -33 to 33 , with higher total scores reflecting greater TA. The HRQ was administered at week two, mid-treatment, and EOT. Participant responses with missing items on the HRQ were excluded from analyses involving HRQ total scores. The HRQ has strong psychometrics and has been shown to correlate with treatment outcome $[29,30]$.

\section{The Eating Disorder Examination (EDE)}

The EDE is a semi-structured investigator-based interview measuring cognitive and behavioral symptoms related to eating disorders [31]. The EDE was used to generate DSM-IV-TR diagnoses for an ED and to assess the severity of symptomatology. Subscales include: 
Weight Concern, Shape Concern, Eating Concern, and Restraint; global scores reflect the overall severity of ED symptoms.

\section{The Anorexia Nervosa Stages of Change Questionnaire (ANSOCQ)}

The ANSOCQ is a 20-item self-report questionnaire assessing a patient's readiness for recovery from AN, with higher total scores reflecting greater readiness for recovery [32].

\section{The Treatment Suitability and Patient Expectations (TSPE)}

The TSPE is a 2-item self-report questionnaire designed to assess a patient's belief regarding her expectation of improvement in her treatment assignment ("How successful do you think your treatment here will be?") and suitability of her assigned treatment. The TSPE items are answered using an 11-point scale ranging from 0 (not at all) to 10 (completely acceptable or extremely suitable). The TSPE is given following the first therapy session, once the patient has met with her therapist and is aware of which treatment to which she has been randomized. The patient is asked to comment on "overall" improvement, rather than improvement on any specific domains (e.g., weight gain, quality of life). The TSPE has been utilized in multiple trials with patients with eating disorders [25, 33-35]. Cronbach's alphas for the TSPE ranged from .75 to .92 over the assessment time points in this trial.

\section{Assessment time points}

The physical assessment, EDE and ANSOCQ were administered at baseline (pre randomization), session 15, and EOT. The HRQ and the TSPE were given following the first therapy session, session 15, and EOT.

\section{Data analysis}

Stepwise multiple regressions were conducted to investigate the predictive utility of patient motivation, believed suitability of treatment, symptom severity, duration of illness, number of previous specialist ED treatment experiences, and BMI on early treatment alliance. Stepwise multiple regressions were also conducted to investigate the predictive power of the variables detailed above, as well as early treatment alliance, on participant ratings of mid and end of treatment alliance. An alpha level of .05 was used to provide maximum power to identify potential predictors of therapeutic alliance.

Post hoc analyses were conducted to better understand the role of significant predictor(s) of TA. An independent $t$-Test was used to examine any differences occurring based on treatment assignment and a pearson correlation was run to understand the predictor(s) relationship to outcome. To determine if a significant meditational effect was present among the significant predictor(s) of TA, TA, and eating disorder symptom outcomes (defined by EDE global score), the methodology outlined by Baron and Kenny was utilized to evaluate if mediation was supported [36].

\section{Results}

A total of 63 participants were randomized to CBT-AN $(n=31)$ or SSCM $(n=32)$. The range of age for study participants was 20-62 $(M=33.4 \pm 9.6)$, with duration of illness ranging from 7 to 49 years $(M=16.6 \pm 8.5)$. The mean BMI for the sample was $16.2(\mathrm{SD}=1.3$, range $=11.8-18.5)$. The majority of participants met criteria for AN restricting subtype $(n=47,74.6 \%)$. No significant differences on any baseline characteristics were found between treatment groups, sites, or group-by-site interactions [25]. Table 1 shows baseline demographics for all predictor variables.

Baseline ratings of how successful participants believed treatment would be was the only variable to significantly predict early $(\beta=0.67, \mathrm{SE}=0.94, p=.01)$, mid $(\beta=0.80$, $\mathrm{SE}=0.80, p=.009)$, and late treatment alliance $(\beta=0.66$, $\mathrm{SE}=1.98, p=.03$; see Table 2 ). Motivation, symptom severity, duration of illness, age, number of previous specialist ED treatment experiences, early therapeutic alliance, and BMI did not significantly predict alliance at any point in treatment ( $p s>.57$; see Table 3 ).

\section{Patient expectations of treatment success}

Participant ratings of anticipated treatment success did not significantly differ based on treatment assignment (CBT-AN $M=7.04 \pm 1.97$, SSCM $M=7.17 \pm 1.56, p=.78$ ). The TSPE item was significantly correlated with EOT $(r(47)=-.40, p=.005)$ and follow-up $(r(44)=-.48, p=.001)$ EDE global scores.

\section{Mediation analyses}

Given the present findings that anticipated treatment success significantly predicted TA, and past findings from this sample indicating TA as a significant predictor of outcome [19], additional analyses were conducted to determine the possibility of a mediating relationship, such that expectations for therapy impacts overall TA, which impacts treatment outcomes (see Fig. 1). Utilizing Baron and Kenny's (1986) four step approach, a full mediation effect is suggested for early TA, such that: step 1) expectations of treatment success (TSPE item 2) significantly predicts EOT symptomology (EDE global score; $p=.005)$; step 2) expectations of treatment success (TSPE item 2) significantly predicts early TA $(p<.001)$; step 3 ) early TA significantly predicts EOT symptomology $(p=.02)$; and step 4$)$ expectations of treatment success no longer significantly predicts EOT symptomology after controlling for early TA $(p=.07)$ [36]. A partial mediation effect is suggested for late TA, such that: step 1) expectations of treatment success significantly predicts EOT symptomology $(p=.005)$; step 2$)$ expectations of 
Table 1 Baseline characteristics of predictor variables for therapeutic alliance

\begin{tabular}{lccc}
\hline Variable & Mean & Standard Deviation & Range (Minimum, Maximum) \\
\hline Age & 33.41 & 9.57 & $42(20,62)$ \\
Duration of Illness & 16.57 & 8.45 & $42(7,49)$ \\
ANSOCQ & 16.57 & 17.89 & $77(0,77)$ \\
BMI & 16.20 & 1.34 & $6.67(11.80,18.47)$ \\
EDE Global Score & 3.10 & 1.31 & $5.26(.41,5.67)$ \\
Frequency of Specialist ED Interventions & 6.80 & 4.16 & $16(2,18)$ \\
TSPE: Suitability of Treatment & 7.41 & 1.78 & $9(1,10)$ \\
TSPE: Success of Treatment & 7.08 & 1.75 & $9(1,10)$
\end{tabular}

Note. $\mathrm{ANSOCQ}=$ The Anorexia Nervosa Stages of Change Questionnaire; BMI = Body Mass Index; EDE $=$ Eating Disorders Examination; ED $=$ Eating Disorders; $\mathrm{TSPE}=$ Treatment Suitability and Patient Expectations

treatment success significantly predicts late TA $(p<.02)$; step 3) late TA significantly predicts EOT symptomology $(p=.002)$; and step 4) expectations of treatment success significantly predicts EOT symptomology after controlling for early TA $(p s=.03)$.

\section{Discussion}

This study examined predictors of TA in a treatment trial offering two psychological therapies for SE-AN. Contrary to our hypotheses, nearly all of the variables examined were not found to be significant predictors of TA at the start of treatment, mid treatment, or EOT. Indeed, patient expectations for treatment, an item in the TSPE which was given following the first therapy session, was found to be the only significant predictor of TA throughout treatment. Mediation analyses suggested that this early treatment factor impacts outcomes such that patient treatment expectations drive the development of TA, which impacts treatment outcomes.

These findings are in contrast to previous research suggesting that increased duration of illness, severity of illness, and decreased motivation may have a negative impact on TA $[3,20-22]$. A number of recent studies confirm the difficulties in engaging individuals with SEAN in psychological treatments [37-39]. This has likely contributed to a prejudice that may be held by many treating clinicians, both in their own expectations for outcome as well as their belief that patients with SE-AN are not interested in positive treatment outcomes. However, it appears that within this sample, illness duration, illness severity, previous failed treatment experiences, and baseline BMI had no significant predictive capacity on patients' therapeutic relationships. While this study did not include patients with a short duration of AN, reported levels of TA in the current study were consistent with the use of the HRQ with other samples with eating disorders [33]. As such, clinicians should remain highly cautious when assuming that illness characteristics can impact the likelihood of forming an engagement. Additionally, this study found that early TA had little predictive validity on later TA ratings. This suggests that when initial impressions are made, other factors may override them and have a greater influence on the strength of the therapeutic relationship as treatment progresses.

Patients' belief in the ability of the treatment to contribute to overall improvement of symptoms was the only significant contributor to their experience of TA at all stages of treatment. This finding is consistent with the literature on adults with $\mathrm{BN}$ [21], and longstanding work noting the importance of hope and alliance in treatments [40]. Further, it suggests that clinicians attempting to engage patients with SE-AN in meaningful psychological work should focus early in treatment on

Table 2 Significant predictors of therapeutic alliance determined through stepwise regression

\begin{tabular}{|c|c|c|c|c|c|c|}
\hline Stepwise regression variable & $\Delta R^{2}$ & $\Delta F$ & $B$ & SE & $B$ & $p$ \\
\hline \multicolumn{7}{|l|}{ Early Treatment Alliance Model } \\
\hline Baseline Rating of Anticipated Treatment Success & .45 & 8.97 & 2.83 & 0.94 & .67 & .01 \\
\hline \multicolumn{7}{|l|}{ Mid Treatment Alliance Model } \\
\hline Baseline Rating of Anticipated Treatment Success & .64 & 12.68 & 2.86 & 0.80 & .80 & .009 \\
\hline \multicolumn{7}{|l|}{ Late Treatment Alliance Model } \\
\hline Baseline Rating of Anticipated Treatment Success & .44 & 7.09 & 5.27 & 1.98 & .66 & .03 \\
\hline
\end{tabular}


Table 3 Variables excluded as significant predictors of therapeutic alliance determined through stepwise regression

\begin{tabular}{|c|c|c|c|}
\hline Stepwise regression variable & Beta In & $t$ & $p$ \\
\hline \multicolumn{4}{|l|}{ Early Treatment Alliance Model } \\
\hline Previous specialist ED intervention & .362 & 1.73 & .12 \\
\hline Baseline ANSOQC Total & .142 & .57 & .58 \\
\hline Duration of illness & -.052 & -.22 & .83 \\
\hline Age & -.026 & -.11 & .92 \\
\hline Baseline BMI & -.173 & -.72 & .49 \\
\hline Baseline rating of treatment suitability & .278 & 1.03 & .33 \\
\hline Baseline EDE Global score & -.039 & -.17 & .87 \\
\hline \multicolumn{4}{|l|}{ Mid Treatment Alliance Model } \\
\hline Previous specialist ED intervention & .119 & .49 & .64 \\
\hline Baseline ANSOQC Total & .366 & 1.64 & .15 \\
\hline Duration of illness & -.353 & -1.70 & .14 \\
\hline Age & -.435 & -1.81 & .12 \\
\hline Baseline BMI & -.403 & -1.63 & .16 \\
\hline Baseline rating of treatment suitability & -.225 & -.74 & .49 \\
\hline Baseline EDE Global score & -.281 & -1.26 & .25 \\
\hline Early Treatment HRQ Total & .087 & .27 & .80 \\
\hline \multicolumn{4}{|l|}{ Late Treatment Alliance Model } \\
\hline Previous specialist ED intervention & -.091 & -.34 & .74 \\
\hline Baseline ANSOQC Total & -.176 & -.61 & .56 \\
\hline Duration of illness & -.229 & -.86 & .41 \\
\hline Age & -.021 & -.07 & .95 \\
\hline Baseline BMI & .300 & .98 & .36 \\
\hline Baseline rating of treatment suitability & -.147 & -.44 & .67 \\
\hline Baseline EDE Global score & -.049 & -.18 & .86 \\
\hline Early Treatment HRQ Total & .205 & .66 & .53 \\
\hline
\end{tabular}

Note. $\mathrm{ED}=$ Eating Disorder; $\mathrm{ANSOQC}=$ The Anorexia Nervosa Stages of Change Questionnaire; $\mathrm{BMI}=$ Body Mass Index; $\mathrm{EDE}=$ Eating Disorders Examination; $\mathrm{HRQ}=$ Helping Relationships Questionnaire bolstering patient expectations about the intervention they are undertaking. This may include providing psychoeducation regarding the efficacy of CBT-AN and SSCM for SE-AN [25], or the benefits of symptom change even when remission is not possible [27]. Where available, these early treatment actions may aid the process of building a positive TA throughout treatment, which in turn supports better outcomes [19, 41]. Indeed, as more research is conducted to identify the most effective means to treat adults with SE-AN, bolstering TA is a likely channel to improve outcomes for currently available treatments [42].

To our knowledge, this is the first study to examine predictors of TA in a sample of SE-AN. Engagement in treatments for SE-AN remains a struggle for clinicians. Therefore, examining predictors of TA as a means to enhance the likelihood of forming a positive TA is crucial. There were several strengths to this study, including assessments with well-validated measures. Despite these strengths, limitations should be considered in the interpretation of these findings. While the sample sizes were sufficient to show differences in outcomes, they were also moderate in size. Further, despite its use across a number of RCTs [25, 33-35], the two-item TSPE remains unvalidated. The TSPE was also administered following the first session, which means it is possible that patient expectations were also influenced by the rapport experienced with the therapist during the first session. For this reason, we are cautious to over interpret these findings until they can be confirmed in future research examining treatment expectation prior to meeting the therapist. Additionally, this sample includes only individuals with SE-AN. While it remains unclear how these results generalize to individuals with a shorter duration or less severe presentations, the authors see this as a future research question, rather than a limitation of the study. It is also of note that the number of analyses conducted increases the risk for Type I error. Future studies should

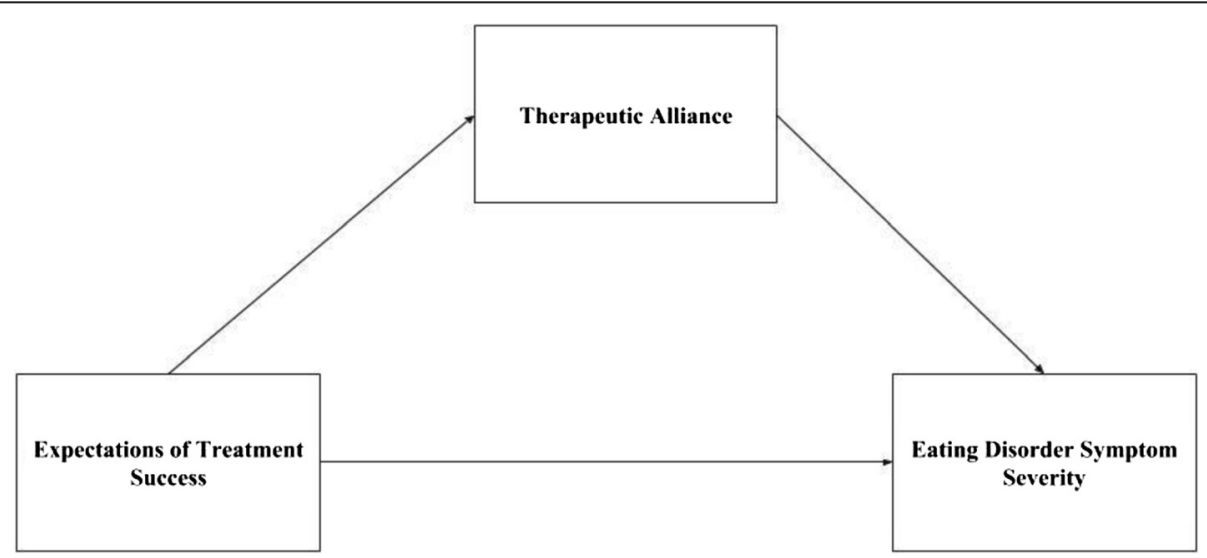

Fig. 1 Therapeutic alliance mediates the relationship between treatment expectations and outcome 
explore predictors of TA in larger samples, including both individuals with $\mathrm{BN}$ and with patients with a shorter duration of AN. This would also exclude the possibility of any floor effects influencing the nonsignificant finding for duration of illness arising from the chronic nature of this group. It may also be beneficial to explore patient characteristics in conjunction with therapist characteristics.

\section{Conclusions}

Results of this study suggest that an initial patient expectation for successful outcome significantly predicts patient-reported TA throughout treatment. Further, a mediational relationship between early patient treatment expectations, TA, and outcomes appears to exist for this sample of adults with SE-AN. Most cases of SE-AN are defined for clinicians by patients' egosyntonic wish to preserve maladaptive behaviors that they identify as having a "functional" purpose [37]. Through this lens, therapeutic relationships can at times be sacrificed, regardless of chronicity. However, recent findings show that positive and strong TA can be established in individuals with SE-AN [19]. The present study expands insight into TA for individuals with SE-AN, demonstrating that TA can be bolstered with early treatment expectations for successful treatment.

\begin{abstract}
Abbreviations
AN: anorexia nervosa; ANSOCQ: anorexia nervosa stages of change questionnaire; BMI: body mass index; BN: bulimia nervosa; CBT-AN: cognitive behavioral therapy for anorexia nervosa; DSM-IV-TR: Diagnostic and Statistical Manual for Mental Disorders, $4^{\text {th }}$ Edition, Text Revision; EDE: eating disorder examination; EOT: end of treatment; HRQ: helping relationships questionnaire; IRB: Institutional review board; RCT: randomized controlled trial; SE-AN: severe and enduring anorexia nervosa; SSCM: specialist supportive clinical management; TA: therapeutic alliance; TSPE: treatment suitability and patient expectations
\end{abstract}

\section{Competing interests}

Daniel Le Grange receives consultant fees from the Training Institute for Child and Adolescent Eating Disorders, LLC. Dr. Le Grange also receives royalties from Guilford Press and Routledge. Stephen Touyz has received fees for serving as consultant to Shire Pharmaceuticals advisory board member. Phillipa Hay receives Honoraria from PLOSMedicine. Stephen Touyz and Phillipa Hay receive royalties from Hogrefe Publications, McGraw Hill Pubs and honoraria from Biomed Central.

\section{Authors' contributions}

CSS and BHB conceived of the study and helped draft the manuscript. ST, $\mathrm{PH}, \mathrm{HL}$, and DLG served as primary investigators of the RCT and helped draft the manuscript. All authors read and approved the final manuscript.

\section{Funding}

Funding for this work was supported by: National Health and Medical Research Council (Australia) PG 457419 (Drs. Touyz, Le Grange, Lacey and Hay), South-west London and St. George's NHS Trust (Dr. Lacey), the Butterfly Foundation (Dr. Touyz), University of Western Sydney (Dr. Hay), and F31 MH106321 (Ms. Stiles-Shields).

\section{Author details}

'Department of Preventive Medicine, Northwestern University Feinberg School of Medicine, Chicago, IL, USA. ${ }^{2}$ The London Centre for Eating Disorders and Body Image, London, UK. ${ }^{3}$ University of Sydney, School of
Psychology, Sydney, Australia. ${ }^{4}$ Department of Psychiatry, University of California, San Francisco, San Francisco, CA, USA. ${ }^{5}$ Department of Pediatrics, University of California, San Francisco, San Francisco, CA, USA. ${ }^{6}$ Centre for Health Research, School of Medicine, Western Sydney University, Sydney, Australia. ${ }^{7}$ St. George's, University of London, Eating Disorders Research Group, London, UK.

Received: 15 February 2016 Accepted: 29 March 2016

Published online: 05 April 2016

\section{References}

1. Bordin E. The generalizability of the psychoanalytic concept of the working alliance. Psychother Theory Res Pract. 1979;16:252-60.

2. Ilardi SS, Craighead WE. The role of nonspecific factors in cognitive-behavior therapy for depression. Clin Psychol Sci Pract. 1994;1:138-55.

3. Macneil C, Hasty M, Evans M, Redlich C, Berk M. The therapeutic alliance: is it necessary or sufficient to engender positive outcomes? Acta Neuropsychiatrica. 2009;2:95-8.

4. Martin D, Garske J, Davis M. Relation of the therapeutic alliance with outcome and other variables: A meta-analytic review. J Consult Clin Psychol. 2000;68:216-20

5. Tasca GA, Lampard AM. Reciprocal influence of alliance to the group and outcome in day treatment for eating disorders. J Counseling Psychol. 2012;59:507.

6. Arnow BA, Steidtmann D, Blasey C, Manber R, Constantino MJ, Klein DN, Markowitz JC, Rothbaum BO, Thase ME, Fisher AJ, Kocsis JH. The relationship between the therapeutic alliance and treatment outcome in two distinct psychotherapies for chronic depression. J Consult Clin Psychol. 2013;81:627.

7. Horvath $A O$, Del Re AC, Fluckiger C, Symonds D. Alliance in individual psychotherapy. Psychother. 2011;48:9-16.

8. Horvath $A O$, Luborsky L. The role of the therapeutic alliance in psychotherapy. J Consult Clin Psychol. 1993;61:561-73.

9. Del Re AC, Flückiger C, Horvath AO, Symonds D, Wampold BE. Therapist effects in the therapeutic alliance-outcome relationship: A restricted-maximum likelihood meta-analysis. Clin Psychol Rev. 2012;32:642-9.

10. Ardito RB, Rabellino D. Therapeutic alliance and outcome of psychotherapy: Historical excursus, measurements, and prospects for research. Front Psychol. 2011;2:270.

11. Brown A, Mountford V, Waller G. Therapeutic alliance and weight gain during cognitive behavioural therapy for anorexia nervosa. Behav Res Ther. 2013:51:216-20.

12. Loeb K, Wilson G, Labouvie E, Pratt E, Hayaki J, Walsh B, et al. Therapeutic alliance and treatment adherence in two interventions for bulimia nervosa: A study of process and outcome. J Consult Clin Psychol. 2005;73:1097-107.

13. Bourion-Bedes S, Baumann C, Kermarrec S, Ligier F, Feillet F, Bonnemains C, Guillemin F, Kabuth B. Prognostic value of early therapeutic alliance in weight recovery: A prospective cohort of 108 adolescents with anorexia nervosa. J Adolesc Health. 2013:52:344-50.

14. Graves TA, N. T, Thompson-Brenner H, Franko DL, Eddy KT, Bourion-Bedes $S$, Brown A, Constantino MJ, Flückiger C, Forsberg S, et al. A Meta-Analysis of the Relation between Therapeutic Alliance and Treatment Outcome in Eating Disorders. Clin Psychol Rev. Under Review.

15. Forsberg S, LoTempio E, Bryson S, Fitzpatrick KK, Le Grange D, Lock J. Therapeutic alliance in two treatments for adolescent anorexia nervosa. Int J Eat Disord. 2013; 46:34-8.

16. Waller $G$, Evans J, Stringer $H$. The therapeutic alliance in the early part of cognitive-behavioral therapy for the eating disorders. Int J Eat Disord. 2012; 45:63-9.

17. Sly R, Morgan JF, Mountford VA, Lacey JH. Predicting premature termination of hospitalised treatment for anorexia nervosa: The roles of therapeutic alliance, motivation, and behaviour change. Eat Behav. 2013;14:119-23.

18. Gallop R, Kennedy SH, Stern D. Therapeutic alliance on an inpatient unit for eating disorders. Int J Eat Disord. 1994;16:405-10.

19. Stiles-Shields C, Touyz S, Hay P, Lacey JH, Crosby RD, Rieger E, Bamford BH, Le Grange D. Therapeutic alliance in two treatments for adults with severe and enduring anorexia nervosa. Int J Eat Disord. 2013;46:783-9.

20. Raue P, Castonguay L, Goldfried M. The working alliance: A comparison of two therapies. Psychother Res. 1993;3:197-207.

21. Constantino M, Arnow B, Blasey C, Agras W. The association between patient characteristics and the therapeutic alliance in cognitive-behavioral 
and interpersonal therapy for bulimia nervosa. J Consult Clin Psychol. 2005; 73:203-11.

22. de la Rie S, Noordenbos G, Donker M, van Furth E. The quality of treatment of eating disorders: a comparison of the therapists' and the patients' perspective. Int J Eat Disord. 2008;41:307-17.

23. Messer S, Wolitzky D, Muran J, Barber J. The therapeutic alliance: An evidence based guide to practice. New York: Guilford Press New York; 2010.

24. Watson JC, Kalogerakos F. The therapeutic alliance in humanistic psychotherapy. In Muran CJ, Barber JP, editors. The therapeutic alliance: An evidence-based guide to practice. New York: Guilford Press; 2010. p. 191-209.

25. Touyz S, Le Grange D, Lacey H, Hay P, Smith R, Maguire S, Bamford B, Pike KM, Crosby RD. Treating severe and enduring anorexia nervosa: a randomized controlled trial. Psychol Med. 2013;43:2501-11.

26. American Psychiatric Association. Diagnostic and statistical manual of mental disorders (DSM-IV-TR). Washington, DC: APA; 2000.

27. Hay PJ, Touyz S, Sud R. Treatment for severe and enduring anorexia nervosa: A review. Aust N Z J Psych. 2012;46:1136-44.

28. Luborsky L. Principles of Psychoanalytic Psychotherapy. New York: Basic Books; 1984.

29. Alexander $L$, Luborsky $L$. The penn helping alliance scales. In: Breenberg $L S$, Pinsoff WM, editors. The Psychotherapeutic Process: A Research Handbook. New York: Guilford Press; 1986. p. 325-66.

30. Luborsky L, McLellan AT, Woody GE, O'Brien CP, Auerbach A. Therapist success and its determinants. Arch Gen Psych. 1985:42:602.

31. Cooper Z, Fairburn CG. The eating disorder examination: a semi-structured interview for the assessment of the specific psychopathology of eating disorders. Int J Eat Disorder. 1987;6:1-8.

32. Rieger E, Touyz $S$, Beumont P. The Anorexia Nervosa Stages of Change Questionnaire (ANSOCQ): information regarding its psychometric properties. Int J Eat Disord. 2002;32:24-38.

33. Zaitsoff SL, Celio Doyle A, Rienecke Hoste R, Le Grange D. How do adolescents with bulimia nervosa rate the acceptability and therapeutic relationship in family-based treatment? Int J Eat Disord. 2008;41:390-8.

34. Le Grange D, Crosby RD, Rathouz PJ, Leventhal BL. A randomized controlled comparison of family-based treatment and supportive psychotherapy for adolescent bulimia nervosa. Arch Gen Psych. 2007;64:1049-56.

35. Lock J, Le Grange D, Agras S, Moye A, Bryson S, Jo B. Randomized clinical trial comparing family-based treatment to adolescent focused therapy for adolescents with anorexia nervosa. Arch Gen Psych. 2010;67:1025-32.

36. Baron RM, Kenny DA. The moderator-mediator variable distinction in social psychological research: Conceptual, strategic, and statistical considerations. J Pers Soc Psychol. 1986;51:1173-82.

37. Strober M. The chronically ill patient with anorexia nervosa: Development, phenomenology and theraputic considerations. In: Grilo CM, Mitchell JM, editors. The Treatment of Eating Disorders. London: The Guildford Press; 2010. p. 225-38

38. Wonderlich S, Mitchell JE, Crosby RD, Myers TC, Kadlec K, LaHaise K, SwanKremeier L, Dokken J, Lange M, Dinkel J. Minimizing and treating chronicity in the eating disorders: a clinical overview. Int J Eat Disord. 2012;45:467-75.

39. Strober M, Johnson C. The need for complex ideas in anorexia nervosa: Why biology, environment, and psyche all matter, why therapists make mistakes, and why clinical benchmarks are needed for managing weight correction. Int J Eat Disord. 2012;45:155-78.

40. Frank JD. Common features of psychotherapy. Aust N Z J Psychiatry. 1972:6:34-40

41. Ackerman SJ, Hilsenroth MJ. A review of therapist characteristics and techniques positively impacting the therapeutic alliance. Clin Psychol Rev. 2003;23:1-33.

42. Crits-Christoph P, Baranackie K, Kurcias J, Beck AT, Carroll K, Perry K, Luborsky L, McLellan A, Woody G, Thompson L. Meta-analysis of therapist effects in psychotherapy outcome studies. Psychother Res. 1991;1:81-91.

\section{Submit your next manuscript to BioMed Central and we will help you at every step:}

- We accept pre-submission inquiries

- Our selector tool helps you to find the most relevant journal

- We provide round the clock customer support

- Convenient online submission

- Thorough peer review

- Inclusion in PubMed and all major indexing services

- Maximum visibility for your research

Submit your manuscript at www.biomedcentral.com/submit
Biomed Central 